\title{
THE ERODING FEELING OF THE ROAD OR CONTROLLING OUR CONVENIENCES WHILE BEING DRIVEN TO DISTRACTION BY PRODIGIOUS INANIMATE OBJECTS THAT KNOW NOT THE PRICE OF PROGRESS
}

Consider our much clichéd love affair with the automobile. Every whiz-bang newfangled feature that crops up in our telephones, computers, TVs, microwave ovens, and institutional carpeting is bound to be inserted into our cars-at a price of course. We know the monetary price is controlled by the production process and the number of units produced, and then by marketing specialists who apply the "whatever-the-market-will-bear" test to determine the maximum price just short of discouraging acceptance. There is, we posit, another fundamental price barrier to unbounded accretion of high tech in autos. Call it the humanity factor. Race car drivers "feel" through their tactile linkages the interface of car and road. A subliminal traction meter controls braking and velocity on the turns. Peripheral vision, familiar sounds, and even intuition locate other cars and dictate when to attempt to pass or get out of the way and when to make an unscheduled pit stop.

Those of us who inhabit only passenger vehicles (with the obvious exception of the Autobahn's merciless Mercedes) must cope in ignominy with the erosion of the feel of the road in face of the onslaught of power-assisted brakes and steering, anti-slip differentials and the newest wrinkle of "wepump-them-for-you" brakes. High tech now threatens to go beyond electric door locks, mirrors, windows, seats, and full Surround Sound ${ }^{(\mathbb{B})}$ stereo amphitheater orchestra-quality compact disc players with complimentary cellular phone and shaving kit. ${ }^{1}$ Soon, high tech will take care of our vehicle opera- tion, our navigation, and perhaps our sightseeing too. For navigating, the Global Positioning System satellite will directly tell our cars where we are so they can take us to our requested destinations; at least we hope they are the ones we requested. ${ }^{2}$ I imagine it's very nice, when bordering on being lost, to know one's precise latitude and longitude on the globe; but in most cases, one already has a vague idea of what country and even what city one is in, and a homing beacon on the closest petrol supply or rest stop would be a tad more useful.

There will also be a Collision Avoidance System on the Intelligent Vehicle Highway intended to help us avoid close encounters of the wrong kind with other vehicles or stationary objects. Many lives will be saved. Nevertheless, one hopes this device can be switched off to allow close approach to neighboring parked cars and meters lest too much acreage be devoted to zerovelocity pursuits.

A simple extrapolation from the threat/counter-threat cycle in defense technology, a source of many civilian widgets, leads us to predict introduction of a stealth auto, able to elude the radar and lidar of various traffic authorities charged with enforcing limits to our speed and other driving irregularities. A nice example of dual-use technology, but every advance has its drawbacks. As you cloak to avoid detection, your neighbor's Collision Avoidance System will blithely drive right through you.

With each augmentation, our five or so senses are further removed from interaction with the environment, and our environment be- comes a safe, very high-tech cocoon. The ultimate question then becomes, "Why take a ride in the car at all?" Let's go all the way to full virtual reality, the ultimate oxymoron. Program your terminal to mount the web and drive you to your destination in the safety of your own home. We can not only dispense with the Collision Avoidance System, but we can experience virtual collisions just for the fun of it. With the appropriate appliances, the feel of the road, not to mention its smell, appearance and taste, will be replicated beyond our ability to discern the difference. What daredevil child now grown who remembers flying by a parent yelling, "look ma, no hands!"3 could resist driving past a favorite haunt yelling, "look guys, no car!"4 I firmly believe that had Surround Sound ${ }^{\circledR}$ been available at the turn of the century, the Model $\mathrm{T}^{\mathbb{B}}$ would have mimicked the reassuring clip-clop sound of a horse-drawn carriage to mitigate the effects of techno-culture shock. The tension between uniquely human qualities and invading technology is no new subject for philosophy. Let's leave the global penetrating perspectives to the philosophers and simply bemoan the increasing rarity of the humanity factor, the real hands-on experience of driving the open road or the city streets. Finally the "auto" in automobile is hitting home.

ELTON N. KaUfMANN

\section{Or makeup case as the case may be.}

2. One can easily imagine a criminal being safely apprehended when police override the suspect's navigational controls.

3. Or " $p a$ " as the case may be.

4. Or "gals" as the case..... 